\title{
Investigation of Aggression Levels of University Students (Kocaeli University Case)
}

\author{
Özlem Keskin ${ }^{1} \Varangle$ \\ Hakan Akdeniz ${ }^{2}$ \\ Faculty of Sports Sciences, Department of Recreation, Kocaeli, Turkey \\ Email:ozlemnht@hotmail.com Tel:+905301171321 \\ Email:hakanakdenia@gmail.com Tel:+905324653736
}

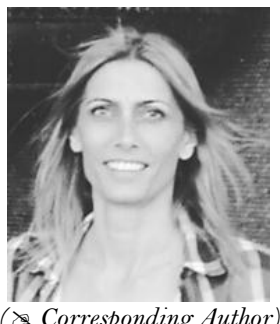

\begin{abstract}
The aim of this study was to investigate the aggression levels of university students in different departments in terms of sport and other variables. The population of the study consists of university students studying at Kocaeli University; the sample group consists of a total of 700 students, 378 male and 322 female, studying in the Faculty of Sports Sciences, Faculty of Communication and Faculty of Education of Kocaeli University. A screening model was used in this survey. A Personal Information form developed by the investigator and a Turkish-adapted Buss-perry aggression scale was utilized to determine the aggressive attitudes of the individuals. The frequency distribution specified the demographic features and analyzed the data; the T-test examined the relationship between the two independent variables; Anova Variance was used to review the connections between more than one variable. Finally, the significance level is accepted as $\mathrm{p}<0.05$. According to the findings of the research, there are statistically significant differences $(\mathrm{p}<0.05)$ in terms of gender, family structure, school department, family attitude, mother still living, sporting situation, kind of sport performed, reason for involvement in sport, cigarettealcohol abuse and income level. We can state that sport positively affects the aggression levels of university students. Sport also varies by the gender variable, and males are more aggressive than females. It can also be pointed out that aggression varies by the school department, family attitude, cigarette-alcohol abuse, mother still living, reason for playing sport and income level.
\end{abstract}

Keywords: Sport, Aggression, University students.

Citation | Özlem Keskin; Hakan Akdeniz (2018). Investigation of Aggression Levels of University Students (Kocaeli University Case). Asian Journal of Education and Training, 4(3): 186-196. History:

Received: 28 March 2018

Revised: 7 May 2018

Accepted: 10 May 2018

Published: 15 May 2018

Licensed: This work is licensed under a Creative Commons

Attribution 3.0 License $(\boldsymbol{c c c}) \mathbf{E}$

Publisher: Asian Online Journal Publishing Group
Contribution/Acknowledgement: Both authors contributed to the conception and design of the study.

Funding: This study received no specific financial support

Competing Interests: The authors declare that they have no conflict of interests.

Transparency: The authors confirm that the manuscript is an honest, accurate, and transparent account of the study was reported; that no vital features of the study have been omitted; and that any discrepancies from the study as planned have been explained.

Ethical: This study follows all ethical practices during writing.

\section{Contents}

1. Introduction

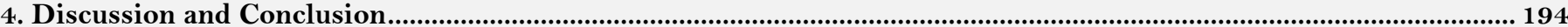

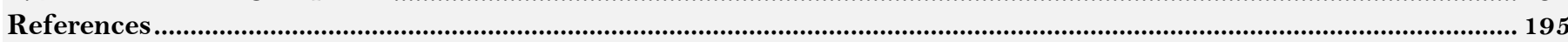

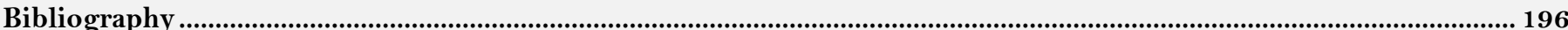




\section{Introduction}

When we think that the existential history of humanity has consisted of various events such as wars, deaths, pillage, and conquest, the place of aggression in the life of a human being can be easily understood. Nowadays, an incremental increase in aggressive behaviors tending towards violence is known around the world (Korkut, 2002; Özdevecioğlu, 2003).

Aggression is a behavior type arising in people in different ways. It is quite difficult to distinguish the reasons that induce this behavior from some of the behavior types because aggression includes destroying, hurting, anger and hate, as well as several verbal reactions. On the other hand, aggression is a concept that is used to express the situations or the attitudes involving different reactions (Cox, 1985; Gergen and Gergen, 1986; Köksal, 1991; Tiryaki, 2000).

Verbal and symbolic behaviors that arise from moods like irritability and tension avoid improvement. Aggression can be exhibited peacefully, can be self-protective and also be harmful behaviors. Aggression is aimed at the people around one, at institutions and at the physical environment as well. One of the reasons put forward for aggression is that the things desired have not gone well or have hit an obstacle. An aggressive attitude can be described as the action-reaction for a feeling of frustration. Some of the aggressive behaviors displayed to eliminate these situations create a feeling of frustration, while some other aggressive actions are affected by maltreatment, being exposed to domestic violence, unkind and punishing child-rearing methods, divorced parents, economic insufficiencies, and social relationship deficiencies (Budak, 2000; Kocacık, 2001; Tok, 2001; Star, 2004; Cüceloğlu, 2005; Leary et al., 2006).

Sports have also been affected by the increase in violence and aggressive events happening in society. An increasing interest in sports, especially football and basketball, and the simultaneous massive increase in sponsorship and advertisement revenue has led to hard competition in these sports branches. As the conditions of the game become harder, the sport arena witnesses vicious conflicts, unethical attitudes, fights and immoralities (Russell, 2003; Özerkan, 2004; Güner, 2006; Kurtic, 2006; Asma, 2008). Sport is not only a struggle to be the most powerful and most successful but also a game, competition, and entertainment that has specific principles and rules. Some athletes or followers who ignore, distort, misemploy or pass off these principles make sport an environment where violence and aggressive behaviors are exhibited (Yetim, 2005).

Aggression in sport can be defined as the athlete, trainer or one or more followers trying to verbally or physically destroy another person by using psychological, social or biological factors and ignoring the universal rules and principles of the sport. There is a need to know the roots of this aggression; trainers, athletes and club managers should take responsibility; media organizations should inhibit such programs that trigger aggression; followers, watchers and society need to be educated within the framework of fair-play to attempt to prevent the violence and aggression (Tiryaki, 2000; Acet, 2005; Dervent, 2007; Erşan et al., 2009; Tutkun et al., 2010)

Aggression has lived in humanity's pocket and also attracted notice as a behavior arising as a result of being affected by events. In this sense, our study was conducted to analyze the aggression levels of the students studying in different departments of universities in terms of sports, sports branches and different demographic variables.

\section{Material and Methods \\ 2.1. Research Method}

This paper was created by using the screening model from quantitative research methods. The screening model describes the global tendencies, attitudes or opinions as quantitative or numerical data via studies on a sample selected from the population. This method is advantageous regarding economy, collecting data quickly and determining the features belonging to a large population by using fewer people (Creswell, 2012).

\subsection{Population and Sample}

A total of 700 students, 378 male and 322 female, who study in the Faculty of Sports Sciences and Faculty of Education in Kocaeli University participated in this survey.

\subsection{Data Collection Tools}

Sociodemographic Information Form: All the students received the socio-demographic information form including the information about Gender, Age, Family Structure, Personal Monthly Income Levels, Mother Still Living, Father Still Living, Smoking Habits, Alcohol Habits, Family Attitudes, Playing Sports, Kind of Sports Played, Weekly Sports Hours and Reason for Playing Sport.

Buss-Perry Aggression Scale: The Buss-Perry aggression scale was developed by Buss and Perry (1992) and adapted to Turkish by Madran (2012). It is a five-point Likert scale that analyzes the aggressive attitudes of university students. This scale consists of 29 items. Nine of these items $(13,8,2,11,25,16,29,22,5)$ constitute physical aggression sub-titles, eight of them $(20,24,3,26,10,15,7,17)$ constitute hostility sub-titles; seven of them $(19,28,1,18,9,23,12)$ constitute anger sub-titles; five of them $(27,6,21,14,4)$ constitute verbal aggression sub-titles. High scores taken from the sub-dimensions of the scale refer to the person's characteristics being high on the relevant dimension. The items of this five-point Likert scale are coded as (1) Absolutely Disagree, (2) Disagree, (3) Neutral, (4) Agree, (5) Absolutely Agree. The ninth and sixteenth items of the scale are inversely coded (Madran, 2012).

\subsection{Analysis of Data}

Frequency distribution was used to analyze the data; a T-test reviewed the relationship between the two independent variables; an Anova Variance analysis test was applied to examine the connections between more than two variables. All these tests were analyzed in SPSS 21, the significance level was accepted as $\mathrm{p}<0.05$. 


\section{Findings}

Table-1. Frequency Table by Socio-Demographic Features

\begin{tabular}{|c|c|c|c|}
\hline & & $\mathbf{n}$ & $\%$ \\
\hline \multirow{2}{*}{ Gender } & Male & 372 & 53,1 \\
\hline & Female & 328 & 46,9 \\
\hline \multirow{3}{*}{ Age } & Under 20 ages & 190 & 27,1 \\
\hline & $20-25$ & 342 & 48,9 \\
\hline & Over 25 ages & 168 & 24,0 \\
\hline \multirow{2}{*}{ Family Structure } & Elementary Family & 489 & 69,9 \\
\hline & Extended Family & 211 & 30,1 \\
\hline \multirow{3}{*}{ School Department } & Faculty of Sport Sciences & 208 & 29,7 \\
\hline & Faculty of Education & 285 & 40,7 \\
\hline & Faculty of Communication & 207 & 29,6 \\
\hline \multirow{4}{*}{ Family Attitude } & Careless & 114 & 16,3 \\
\hline & Democratic & 189 & 27,0 \\
\hline & Authoritative & 188 & 26,9 \\
\hline & Nurturing & 209 & 29,9 \\
\hline \multirow{2}{*}{ Aliveness of Mother } & Yes & 569 & 81,3 \\
\hline & No & 131 & 18,7 \\
\hline \multirow{2}{*}{ Aliveness of Father } & Yes & 569 & 81,3 \\
\hline & No & 131 & 18,7 \\
\hline \multirow{3}{*}{ Personally Monthly Income Level } & Between $0-1000$ & 174 & 24,9 \\
\hline & Between 1000-3000 & 342 & 48,9 \\
\hline & 3000 and over & 184 & 26,3 \\
\hline \multirow{2}{*}{ Smoking Habit } & Yes & 337 & 48,1 \\
\hline & No & 363 & 51,9 \\
\hline \multirow{2}{*}{ Alcohol Habit } & Yes & 248 & 35,4 \\
\hline & No & 452 & 64,6 \\
\hline \multirow{2}{*}{ Sport Situation } & Yes & 392 & 54,6 \\
\hline & No & 308 & 45,4 \\
\hline \multirow{3}{*}{ Kind of Sport } & Personal & 239 & 34,1 \\
\hline & Team & 153 & 21,9 \\
\hline & None & 308 & 44,0 \\
\hline \multirow{4}{*}{ Weekly Sports Hour } & $\mathrm{O}-1$ & 47 & 6,7 \\
\hline & $2-4$ & 196 & 28,0 \\
\hline & $4-7$ & 153 & 21,9 \\
\hline & None & 304 & 43,4 \\
\hline \multirow{4}{*}{ Reason to Sport } & Physical & 119 & 17,0 \\
\hline & Health & 114 & 16,3 \\
\hline & Physical Health & 149 & 21,3 \\
\hline & None & 318 & 45,4 \\
\hline
\end{tabular}

As is seen in Table 1, 328 of the attendees were females, 372 of them were males. The frequency distributions of the attendees by the departments are found as 208 for the faculty of sports sciences; 285 for the faculty of education; 207 for the faculty of communication.

\begin{tabular}{|c|c|c|c|c|c|c|}
\hline & Gender & n & $\bar{x}$ & $\pm \mathrm{ss}$ & $t$ & p \\
\hline \multirow{2}{*}{ Physical aggression } & Male & 372 & 30,91 & 6,41 & \multirow{2}{*}{2,405} & \multirow{2}{*}{,016* } \\
\hline & Female & 328 & 29,72 & 6,52 & & \\
\hline \multirow{2}{*}{ Hostility } & Male & 372 & 27,92 & 5,93 & \multirow{2}{*}{2,201} & \multirow{2}{*}{,028* } \\
\hline & Female & 328 & 26,95 & 5,64 & & \\
\hline \multirow{2}{*}{ Anger } & Male & 372 & 24,50 & 4,84 & \multirow{2}{*}{3,513} & \multirow{2}{*}{,000* } \\
\hline & Female & 328 & 23,22 & 4,72 & & \\
\hline \multirow{2}{*}{ Aggression } & Male & 372 & 17,14 & 3,48 & \multirow{2}{*}{394} & \multirow{2}{*}{,693 } \\
\hline & Female & 328 & 17,03 & 3,60 & & \\
\hline \multirow{2}{*}{ Total Aggression } & Male & 372 & 97,14 & 17,63 & \multirow{2}{*}{2,970} & \multirow{2}{*}{,003* } \\
\hline & Female & 328 & 93,08 & 17,57 & & \\
\hline
\end{tabular}

In Table 2, a significant difference is seen between male and female when looking at the sub-scales of aggression levels by the Gender variable. A significant difference is also observed between the male and female in Hostility sub-scale. One other significant difference $(\mathrm{P}<0,05)$ can be seen between male and female in Anger subscale. Total aggression scale has a significant difference $(\mathrm{P}<0,05)$ as well. There is no significant difference between the genders in Verbal Aggression sub-scale 
Table-3. Significance Table of Aggression Levels Sub-Scales by the Department Variable.

\begin{tabular}{|c|c|c|c|c|c|c|c|}
\hline Your Department & & Gender & n & $\mathbf{x}$ & sd & $\mathbf{t}$ & p \\
\hline \multirow{10}{*}{ Faculty of Sports Sciences } & \multirow{2}{*}{ Physical Aggression } & Male & 109 & 28,96 & 7,85 & \multirow{2}{*}{, 815} & \multirow{2}{*}{, 416} \\
\hline & & Female & 99 & 28,06 & 8,00 & & \\
\hline & \multirow{2}{*}{ Hostility } & Male & 109 & 26,53 & 6,62 & \multirow{2}{*}{, 012} & \multirow{2}{*}{, 990} \\
\hline & & Female & 99 & 26,52 & 6,34 & & \\
\hline & \multirow{2}{*}{ Anger } & Male & 109 & 23,42 & 5,39 & \multirow{2}{*}{, 762} & \multirow{2}{*}{, 447} \\
\hline & & Female & 99 & 22,87 & 4,82 & & \\
\hline & \multirow{2}{*}{ Verbal Aggression } & Male & 109 & 16,67 & 3,60 & \multirow{2}{*}{, 145} & \multirow{2}{*}{, 885} \\
\hline & & Female & 99 & 16,60 & 4,04 & & \\
\hline & \multirow{2}{*}{ Total Aggression } & Male & 109 & 92,34 & 20,60 & \multirow{2}{*}{, 626} & \multirow{2}{*}{, 532} \\
\hline & & Female & 99 & 90,51 & 20,59 & & \\
\hline \multirow{10}{*}{ Faculty of Education } & \multirow{2}{*}{ Physical Aggression } & Male & 109 & 31,78 & 5,04 & \multirow{2}{*}{1,18} & \multirow{2}{*}{, 237} \\
\hline & & Female & 99 & 31,07 & 4,98 & & \\
\hline & \multirow{2}{*}{ Hostility } & Male & 109 & 28,17 & 5,01 & \multirow{2}{*}{1,42} & \multirow{2}{*}{, 156} \\
\hline & & Female & 99 & 27,32 & 4,97 & & \\
\hline & \multirow{2}{*}{ Anger } & Male & 109 & 25,16 & 4,13 & \multirow{2}{*}{2,90} & \multirow{2}{*}{,oO4* } \\
\hline & & Female & 99 & 23,71 & 4,21 & & \\
\hline & \multirow{2}{*}{ Verbal Aggression } & Male & 109 & 17,40 & 3,35 & \multirow{2}{*}{,009 } & \multirow{2}{*}{, 993} \\
\hline & & Female & 99 & 17,40 & 3,07 & & \\
\hline & \multirow{2}{*}{ Total Aggression } & Male & 109 & 99,19 & 14,26 & & Leg*⿻丷木 \\
\hline & & Female & 99 & 95,57 & 14,17 & 2,10 & ,000 \\
\hline & Phrcical A rorreccion & Male & 109 & 31,66 & 6,17 & 091 & ogo* \\
\hline & t & Female & 99 & 29,62 & 6,32 & 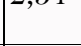 & |, \\
\hline & Hostility & Male & 109 & 28,94 & 6,23 & & $015 *$ * \\
\hline & Hostminy & Female & 99 & 26,88 & 5,76 & $2,+5$ & ,010 \\
\hline Communication & $A n m e r$ & Male & 109 & 24,65 & 5,04 & & \\
\hline - & 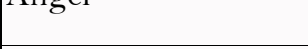 & Female & 99 & 22,91 & 5,23 & ],09 & ,01 \\
\hline & Verbal A orression & Male & 109 & 17,22 & 3,54 & 70 & 1600 \\
\hline & 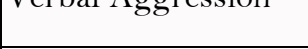 & Female & 99 & 16,97 & 3,76 & , & ], \\
\hline & & Male & 109 & 99,01 & 18,03 & & \\
\hline & 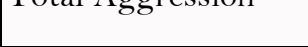 & Female & 99 & 92,35 & 18,11 & 2,00 & |, \\
\hline
\end{tabular}

*p<0,05

As is seen in Table 3, when looking at the sub-scales of aggression levels by the gender and department, there is a significant difference between male and females in Anger and Total Aggression sub-scales in the faculty of education. A significant difference $(\mathrm{P}<0,05)$ is observed in Physical Aggression, Hostility, Anger and Total Aggression sub-scales in the faculty of communication. No significant difference is found in other research groups.

Table-4. Table of Aggression Levels Sub-Scales by Family Structure

\begin{tabular}{|c|c|c|c|c|c|c|}
\hline Family Structure & & $\mathbf{n}$ & $\mathbf{x}$ & sd & $\mathbf{t}$ & $\mathbf{p}$ \\
\hline \multirow{2}{*}{ Physical aggression } & Elementary Family & 489 & 30,00 & 6,33 & \multirow{2}{*}{$-2,190$} & \multirow{2}{*}{, $029^{*}$} \\
\hline & Extended Family & 211 & 31,18 & 6,78 & & \\
\hline \multirow{2}{*}{ Hostility } & Elementary Family & 489 & 27,20 & 5,66 & \multirow{2}{*}{$-1,850$} & \multirow{2}{*}{,065 } \\
\hline & Extended Family & 211 & 28,10 & 6,13 & & \\
\hline \multirow{2}{*}{ Anger } & Elementary Family & 489 & 23,60 & 4,73 & \multirow{2}{*}{$-2,546$} & \multirow{2}{*}{, $011^{*}$} \\
\hline & Extended Family & 211 & 24,61 & 4,97 & & \\
\hline \multirow{2}{*}{ Verbal Aggression } & Elementary Family & 489 & 16,93 & 3,45 & \multirow{2}{*}{$-1,799$} & \multirow{2}{*}{, 072} \\
\hline & Extended Family & 211 & 17,45 & 3,70 & & \\
\hline \multirow{2}{*}{ Total Aggression } & Elementary Family & 489 & 94,19 & 17,18 & \multirow{2}{*}{$-2,389$} & \multirow{2}{*}{, $017^{*}$} \\
\hline & Extended Family & 211 & 97,75 & 18,68 & & \\
\hline
\end{tabular}

When looking at aggression level sub-scale of the family structure, since the Physical Aggression is $\mathrm{p}=0,029<0,049$ and Anger are $\mathrm{p}=0,011<0,049$, there is a significant difference between the Family Structures. Since $\mathrm{p}>0,049$, there is no significant difference between Hostility and Verbal Aggression. 
Table-5. Significance Table of Aggression Levels Sub-Scales by Department Variable.

\begin{tabular}{|c|c|c|c|c|c|c|c|}
\hline \multicolumn{2}{|c|}{ School Department } & & \multirow{2}{*}{$\frac{\mathbf{x}}{28,53}$} & \multirow{2}{*}{$\frac{\text { sd }}{7,92}$} & \multirow{5}{*}{$\begin{array}{l}\mathbf{t} \\
12,91\end{array}$} & \multirow{5}{*}{\begin{tabular}{|l|}
$\mathbf{p}$ \\
, $000^{*}$
\end{tabular}} & \multirow{5}{*}{\begin{tabular}{|l} 
Significance \\
Faculty of Sport Sciences - \\
Faculty of Education \\
Faculty of Sport Sciences - \\
Faculty of Communication
\end{tabular}} \\
\hline \multirow{4}{*}{$\begin{array}{l}\text { Physical } \\
\text { Aggression }\end{array}$} & Faculty of Sport Sciences & & & & & & \\
\hline & Faculty of Education & 285 & 31,46 & 5,01 & & & \\
\hline & Communication & 207 & 30,69 & 6,30 & & & \\
\hline & Total & 700 & 30,36 & 6,48 & & & \\
\hline \multirow{4}{*}{ Hostility } & Faculty of Sport Sciences & 208 & 26,53 & 6,47 & \multirow{4}{*}{3,81} & \multirow{4}{*}{, $023^{*}$} & \multirow{4}{*}{$\begin{array}{l}\text { Faculty of Sport Sciences- } \\
\text { Faculty of Communication }\end{array}$} \\
\hline & Faculty of Education & 285 & 27,79 & 5,00 & & & \\
\hline & Communication & 207 & 27,96 & 6,08 & & & \\
\hline & Total & 700 & 27,47 & 5,81 & & & \\
\hline \multirow{4}{*}{ Anger } & Faculty of Sport Sciences & 208 & 23,16 & 5,12 & \multirow{4}{*}{4,70} & \multirow{4}{*}{, $009 *$} & \multirow{4}{*}{$\begin{array}{l}\text { Faculty of Sport Sciences- } \\
\text { Faculty of Education }\end{array}$} \\
\hline & Faculty of Education & 285 & 24,50 & 4,23 & & & \\
\hline & Communication & 207 & 23,82 & 5,19 & & & \\
\hline & Total & 700 & 23,90 & 4,82 & & & \\
\hline \multirow{4}{*}{$\begin{array}{l}\text { Verbal } \\
\text { Aggression }\end{array}$} & Faculty of Sport Sciences & 208 & 16,64 & 3,80 & \multirow{4}{*}{2,79} & \multirow{4}{*}{,062 } & \multirow{4}{*}{$\begin{array}{l}\text { Faculty of Sport Sciences- } \\
\text { Faculty of Education }\end{array}$} \\
\hline & Faculty of Education & 285 & 17,40 & 3,22 & & & \\
\hline & Communication & 207 & 17,10 & 3,64 & & & \\
\hline & Total & 700 & 17,09 & 3,53 & & & \\
\hline \multirow{4}{*}{$\begin{array}{l}\text { Total } \\
\text { Aggression }\end{array}$} & Faculty of Sport Sciences & 208 & 91,47 & 20,57 & \multirow{4}{*}{7,11} & \multirow{4}{*}{, $001 *$} & \multirow{4}{*}{$\begin{array}{l}\text { Faculty of Sport Sciences - } \\
\text { Faculty of Education } \\
\text { Faculty of Sport Sciences - } \\
\text { Communication Faculty }\end{array}$} \\
\hline & Faculty of Education & 285 & 97,56 & 14,31 & & & \\
\hline & Communication & 207 & 95,89 & 18,33 & & & \\
\hline & Total & 700 & 95,26 & 17,70 & & & \\
\hline
\end{tabular}

In Table 5, when looking at the aggression levels sub-scales by departments, a significant difference $(\mathrm{p}<0,05)$ is seen between faculty of sports sciences and faculty of communication and also between faculty of sports sciences and faculty of education in Physical Aggression sub-scale. One more significant difference $(p<0,05)$ can be observed between faculty of sports sciences and faculty of communication in Hostility sub-scale. There is a significant difference $(p<0,05)$ between the faculty of sports sciences and faculty of education in Anger sub-scale. A significant difference $(p<0,05)$ is observed between faculty of sport sciences and faculty of communication and also between faculty of sports sciences and faculty of education in Total aggression scores.

\begin{tabular}{|c|c|c|c|c|c|c|c|}
\hline Family Attitude & & n & $\mathbf{x}$ & sd & $t$ & $\mathbf{p}$ & Significance \\
\hline \multirow{5}{*}{$\begin{array}{l}\text { Physical } \\
\text { Aggression }\end{array}$} & Careless & 114 & 30,3243 & 6,77047 & \multirow{5}{*}{5,156} & \multirow{5}{*}{, $002 *$} & \multirow{5}{*}{$\begin{array}{l}\text { Democratic - Nurturing, } \\
\text { Authoritative - Nurturing }\end{array}$} \\
\hline & Democratic & 189 & 30,8075 & 6,32501 & & & \\
\hline & Authoritative & 188 & 31,4565 & 4,54946 & & & \\
\hline & \begin{tabular}{|l} 
Nurturing \\
\end{tabular} & 209 & 29,0000 & 7,65729 & & & \\
\hline & Total & 700 & 30,3619 & 6,48937 & & & \\
\hline \multirow{5}{*}{ Hostility } & \begin{tabular}{|l|} 
Careless \\
\end{tabular} & 114 & 27,4071 & 5,90193 & \multirow{5}{*}{2,054} & \multirow{5}{*}{, 105} & \\
\hline & Democratic & 189 & 27,3422 & 6,38887 & & & \\
\hline & Authoritative & 188 & 28,3135 & 4,69726 & & & \\
\hline & \begin{tabular}{|l|} 
Nurturing \\
\end{tabular} & 209 & 26,8719 & 6,09361 & & & \\
\hline & Total & 700 & 27,4753 & 5,81890 & & & \\
\hline \multirow{5}{*}{ Anger } & Careless & 114 & 23,6460 & 5,01412 & \multirow{5}{*}{1,712} & \multirow{5}{*}{, 163} & \\
\hline & Democratic & 189 & 23,7923 & 4,93034 & & & \\
\hline & Authoritative & 188 & 24,5691 & 3,97862 & & & \\
\hline & Nurturing & 209 & 23,5481 & 5,28672 & & & \\
\hline & Total & 700 & 23,9061 & 4,82770 & & & \\
\hline \multirow{5}{*}{ Verbal Aggression } & Careless & 114 & 17,1593 & 3,93602 & \multirow{5}{*}{1,064} & \multirow{5}{*}{,364 } & \\
\hline & Democratic & 189 & \begin{tabular}{|l|}
17,1337 \\
\end{tabular} & 3,54363 & & & \\
\hline & Authoritative & 188 & 17,3797 & 2,98026 & & & \\
\hline & Nurturing & 209 & 16,7548 & 3,76129 & & & \\
\hline & Total & 700 & 17,0906 & 3,53885 & & & \\
\hline \multirow{5}{*}{ Total Aggression } & Careless & 114 & 94,2407 & 19,27706 & \multirow{5}{*}{3,251} & \multirow{5}{*}{, $021 *$} & \multirow{5}{*}{ Nurturing - Authoritative } \\
\hline & Democratic & 189 & 95,5587 & 18,27077 & & & \\
\hline & Authoritative & 188 & 98,3278 & 12,98552 & & & \\
\hline & Nurturing & 209 & 92,8109 & 19,59219 & & & \\
\hline & Total & 700 & 95,2650 & 17,70845 & & & \\
\hline
\end{tabular}

As is seen in Table 6, when looking at aggression levels sub-scales by the family attitude variable, there is seen a significant difference between democratic - nurturing and also between authoritative and nurturing in Physical aggression sub-scale. A significant difference $(\mathrm{P}<0,05)$ is observed between Nurturing and Authoritative when looking at total aggression score. 
Table-7. Significance Table of Aggression Levels Sub Scales by Aliveness of Mother Variable

\begin{tabular}{|c|c|c|c|c|c|c|}
\hline \multicolumn{2}{|l|}{ Is your mother alive? } & $\mathbf{n}$ & $\mathbf{x}$ & sd & $\mathrm{t}$ & $\mathbf{p}$ \\
\hline \multirow{2}{*}{ Physical aggression } & Yes & 569 & 30,0071 & 6,45252 & \multirow{2}{*}{$-3,017$} & \multirow{2}{*}{, $003^{*}$} \\
\hline & No & 131 & 31,9141 & 6,44666 & & \\
\hline \multirow{2}{*}{ Hostility } & Yes & 569 & 27,1577 & 5,77062 & \multirow{2}{*}{$-2,983$} & \multirow{2}{*}{,003* } \\
\hline & No & 131 & 28,8385 & 5,85060 & & \\
\hline \multirow{2}{*}{ Anger } & Yes & 569 & 23,6014 & 4,77193 & \multirow{2}{*}{$-3,479$} & \multirow{2}{*}{, $001 *$} \\
\hline & No & 131 & 25,2231 & 4,86447 & & \\
\hline \multirow{2}{*}{ Verbal Aggression } & Yes & 569 & 16,8619 & 3,36103 & \multirow{2}{*}{$-3,582$} & \multirow{2}{*}{, $000^{*}$} \\
\hline & No & 131 & 18,0846 & 4,09393 & & \\
\hline \multirow{2}{*}{ Total Aggression } & Yes & 569 & 94,2099 & 17,32903 & \multirow{2}{*}{$-3,232$} & \multirow{2}{*}{, $001^{*}$} \\
\hline & No & 131 & 99,8480 & 18,65954 & & \\
\hline
\end{tabular}

*p<0,05

There is seen a significant difference between yes and no in physical aggression sub-scale when looking at aggression levels sub-scales by their 'aliveness of mother' status. A significant difference is seen between yes and no in Hostility sub-scale. There is the significant difference between yes and no in Anger sub-scale. Verbal aggression sub-scale has the significant difference between yes and no. There is seen significant difference $(p<0,05)$ between yes and no in total aggression scores.

Table-8. Significance Table of Aggression Levels Sub Scales by Aliveness of Father Variable

\begin{tabular}{|c|c|c|c|c|c|c|}
\hline Is your father alive? & & $\mathbf{n}$ & $\mathbf{x}$ & sd & $\mathbf{t}$ & $\mathbf{p}$ \\
\hline \multirow{2}{*}{ Physical aggression } & Yes & 569 & 30,2890 & 6,52878 & \multirow{2}{*}{,- 628} & \multirow{2}{*}{, 530} \\
\hline & No & 131 & 30,6935 & 6,32221 & & \\
\hline \multirow{2}{*}{ Hostility } & Yes & 569 & 27,3476 & 5,75996 & \multirow{2}{*}{$-1,210$} & \multirow{2}{*}{, 227} \\
\hline & No & 131 & 28,0394 & 6,06369 & & \\
\hline \multirow{2}{*}{ Anger } & Yes & 569 & 23,7815 & 4,84054 & \multirow{2}{*}{$-1,419$} & \multirow{2}{*}{, 156} \\
\hline & No & 131 & 24,4496 & 4,75158 & & \\
\hline \multirow{2}{*}{ Verbal Aggression } & Yes & 569 & 17,0071 & 3,49365 & \multirow{2}{*}{$-1,298$} & \multirow{2}{*}{, 195} \\
\hline & No & 131 & 17,4538 & 3,72111 & & \\
\hline \multirow{2}{*}{ Total Aggression } & Yes & 569 & 94,9418 & 17,61013 & \multirow{2}{*}{$-1,018$} & \multirow{2}{*}{,309 } \\
\hline & No & 131 & 96,7712 & 18,15998 & & \\
\hline
\end{tabular}
$\overline{\mathrm{p}>0,05}$

There is no significant difference ( $\mathrm{p}>0,05)$ between physical aggression, hostility, anger, verbal aggression subscales and total aggression scores when looking at aggression levels sub-scales by aliveness of mothers variable.

Table-9. Significance Table of Aggression Levels Sub Scales by Playing Sports Variable

\begin{tabular}{|c|c|c|c|c|c|c|}
\hline Do you play sports? & & $\mathbf{n}$ & $\mathbf{x}$ & sd & $\mathbf{t}$ & $\mathbf{p}$ \\
\hline \multirow{2}{*}{ Physical aggression } & Yes & 382 & 29,02 & 6,72 & \multirow{2}{*}{$-6,008$} & \multirow{2}{*}{,OOO* } \\
\hline & No & 318 & 31,93 & 5,82 & & \\
\hline \multirow{2}{*}{ Hostility } & Yes & 382 & 26,49 & 5,98 & \multirow{2}{*}{$-4,970$} & \multirow{2}{*}{, $000^{*}$} \\
\hline & No & 318 & 28,66 & 5,38 & & \\
\hline \multirow{2}{*}{ Anger } & Yes & 382 & 23,03 & 4,87 & \multirow{2}{*}{$-5,313$} & \multirow{2}{*}{, $000^{*}$} \\
\hline & No & 318 & 24,96 & 4,55 & & \\
\hline \multirow{2}{*}{ Verbal Aggression } & Yes & 382 & 16,37 & 3,59 & \multirow{2}{*}{$-5,963$} & \multirow{2}{*}{, $000^{*}$} \\
\hline & No & 318 & 17,94 & 3,27 & & \\
\hline \multirow{2}{*}{ Total Aggression } & Yes & 382 & 91,54 & 18,22 & \multirow{2}{*}{$-6,159$} & \multirow{2}{*}{, $000^{*}$} \\
\hline & No & 318 & 99,80 & 15,95 & & \\
\hline
\end{tabular}

In Table 9 , there is seen a significant difference $(\mathrm{p}<0,05)$ between yes and no in physical aggression sub-scale when looking at aggression levels sub-scales by playing sports status. A significant difference $(p<0,05)$ is also observed between yes and no in Hostility sub-scale. There is a significant difference $(p<0,05)$ between yes and no in Anger sub-scale. A significant difference $(\mathrm{p}<0,05)$ is also seen between yes and no in Verbal aggression sub-scale. We can see the significant difference $(\mathrm{p}<0,05)$ between yes and no in Total aggression sub-scale. 
Table-10. Significance Table of Aggression Levels Sub Scales by Type of Sports Played Variable

\begin{tabular}{|c|c|c|c|c|c|c|c|}
\hline Do you play sports? & & $\mathbf{n}$ & $\mathbf{x}$ & sd & $\mathbf{t}$ & p & Significance \\
\hline \multirow{4}{*}{ Physical aggression } & Individual & 239 & 28,62 & 7,00 & \multirow{4}{*}{28,483} & \multirow{4}{*}{, $000 *$} & \multirow{4}{*}{$\begin{array}{l}\text { Individual - None } \\
\text { Team- None }\end{array}$} \\
\hline & Team & 153 & 28,97 & 6,64 & & & \\
\hline & None & 308 & 32,37 & 5,36 & & & \\
\hline & Total & 700 & 30,36 & 6,48 & & & \\
\hline \multirow{4}{*}{ Hostility } & Individual & 239 & 26,05 & 6,15 & \multirow{4}{*}{18,561} & \multirow{4}{*}{, $000^{*}$} & \multirow{4}{*}{$\begin{array}{l}\text { Individual - None } \\
\text { Team-None }\end{array}$} \\
\hline & Team & 153 & 26,77 & 6,00 & & & \\
\hline & None & 308 & 28,94 & 5,08 & & & \\
\hline & Total & 700 & 27,47 & 5,81 & & & \\
\hline \multirow{4}{*}{ Anger } & Individual & 239 & 22,70 & 4,97 & \multirow{4}{*}{17,776} & \multirow{4}{*}{, $000 *$} & \multirow{4}{*}{ Individual-None, Team-None } \\
\hline & Team & 153 & 23,45 & 4,89 & & & \\
\hline & None & 308 & 25,07 & 4,40 & & & \\
\hline & Total & 700 & 23,90 & 4,82 & & & \\
\hline \multirow{4}{*}{ Verbal Aggression } & Individual & 239 & 16,41 & 3,53 & \multirow{4}{*}{20,727} & \multirow{4}{*}{, $\mathrm{OOO}^{*}$} & \multirow{4}{*}{ Individual-None Team-None } \\
\hline & Team & 153 & 16,24 & 3,68 & & & \\
\hline & None & 308 & 18,03 & 3,24 & & & \\
\hline & Total & 700 & 17,09 & 3,53 & & & \\
\hline \multirow{4}{*}{ Total Aggression } & Individual & 239 & 90,24 & 18,59 & \multirow{4}{*}{26,155} & \multirow{4}{*}{, $000 *$} & \multirow{4}{*}{ Individual - None Team-None } \\
\hline & Team & 153 & 92,50 & 18,28 & & & \\
\hline & None & 308 & 100,60 & 15,08 & & & \\
\hline & Total & 700 & 95,26 & 17,70 & & & \\
\hline
\end{tabular}

As is seen Table 10, when looking at aggression levels sub-scales by the 'type of sport performed' variable, there is a significant difference $(\mathrm{p}<0,05)$ between individual and none and also between the team and none in Physical aggression sub-scale. A significant difference $(p<0,05)$ is seen between individual and none and also between the team and none in Hostility sub-scale. A significant difference $(p<0,05)$ is seen between individual and none and also between the team and none in Anger sub-scale. There is a significant difference $(\mathrm{p}<0,05)$ between individual and none and also between the team and none in Verbal aggression sub-scale. When looking at total aggression scores, there is seen a significant difference $(\mathrm{P}<0,05)$ between individual and none and also between team and none.

Table-11. Significance Table of Aggression Levels Sub Scales by Reason to Sport Variable

\begin{tabular}{|c|c|c|c|c|c|c|c|}
\hline Reason to Sport & & $n$ & $\mathbf{x}$ & sd & $\mathbf{t}$ & p & Significance \\
\hline \multirow{5}{*}{$\begin{array}{l}\text { Physical } \\
\text { Aggression }\end{array}$} & Physical & 119 & 29,15 & 6,63 & \multirow{5}{*}{22,785} & \multirow{5}{*}{,OOO* } & \multirow{5}{*}{$\begin{array}{l}\text { Physical - None } \\
\text { Health - None } \\
\text { Physical health - None }\end{array}$} \\
\hline & Health & 114 & 27,36 & 8,84 & & & \\
\hline & Physical Health & 149 & 29,28 & 5,08 & & & \\
\hline & None & 318 & 32,37 & 5,30 & & & \\
\hline & Total & 700 & 30,36 & 6,48 & & & \\
\hline \multirow{5}{*}{ Hostility } & Physical & 119 & 26,86 & 5,85 & \multirow{5}{*}{15,535} & \multirow{5}{*}{,OOO* } & \multirow{5}{*}{$\begin{array}{l}\text { Physical - None } \\
\text { Health - None } \\
\text { Physical Health - None }\end{array}$} \\
\hline & Health & 114 & 25,34 & 7,67 & & & \\
\hline & Physical Health & 149 & 26,31 & 4,73 & & & \\
\hline & None & 318 & 29,01 & 5,07 & & & \\
\hline & Total & 700 & 27,47 & 5,81 & & & \\
\hline \multirow{5}{*}{ Anger } & Physical & 119 & 23,50 & 4,84 & \multirow{5}{*}{15,100} & \multirow{5}{*}{,OOO* } & \multirow{5}{*}{$\begin{array}{l}\text { Physical - None } \\
\text { Health - None } \\
\text { Physical Health - None }\end{array}$} \\
\hline & Health & 114 & 22,20 & 6,32 & & & \\
\hline & Physical Health & 149 & 22,85 & 3,69 & & & \\
\hline & None & 318 & 25,15 & 4,35 & & & \\
\hline & Total & 700 & 23,90 & 4,827 & & & \\
\hline \multirow{5}{*}{ Verbal Aggression } & Physical & 119 & 16,37 & 3,81 & \multirow{5}{*}{15,054} & \multirow{5}{*}{,000* } & \multirow{5}{*}{$\begin{array}{l}\text { Physical - None } \\
\text { Health - None } \\
\text { Physical Health - None }\end{array}$} \\
\hline & Health & 114 & 16,09 & 4,34 & & & \\
\hline & Physical Health & 149 & 16,38 & 2,66 & & & \\
\hline & None & 318 & 18,04 & 3,24 & & & \\
\hline & Total & 700 & 17,09 & 3,53 & & & \\
\hline \multirow{5}{*}{ Total Aggression } & Physical & 119 & 92,30 & 18,31 & \multirow{5}{*}{20,784} & \multirow{5}{*}{,OOO* } & \multirow{5}{*}{$\begin{array}{l}\text { Physical - None } \\
\text { Health - None } \\
\text { Physical Health - None }\end{array}$} \\
\hline & Health & 114 & 88,08 & 24,12 & & & \\
\hline & Physical Health & 149 & 91,41 & 13,03 & & & \\
\hline & None & 318 & 100,79 & 14,92 & & & \\
\hline & Total & 700 & 95,26 & 17,70 & & & \\
\hline
\end{tabular}


As is seen Table 11, when looking at aggression levels sub-scales by 'reason to sport' variable, a significant difference $(\mathrm{p}<0,05)$ is seen between health and none and also between physical health and none in Physical aggression sub-scale. There is seen a significant difference $(\mathrm{p}<0,05)$ between physical and none, between health and none and also between physical health and none in Hostility sub-scale. A significant difference $(p<0,05)$ is observed between physical and none, between health and none and also between physical health and none in Anger sub-scale. A significant difference $(p<0,05)$ can be seen between physical and none, between health and none and also between physical health and none in Verbal aggression sub-scale. There is seen a significant difference $(\mathrm{p}<0,05)$ between physical and none, between health and none and also between physical health and none in Total aggression subscale.

Table-12. Significance Table of Aggression Levels Sub Scales by Smoking Variable (T-test)

\begin{tabular}{|c|c|c|c|c|c|c|}
\hline Do you smoke? & & n & $\mathbf{x}$ & sd & $t$ & 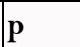 \\
\hline \multirow{2}{*}{ Physical aggression } & Yes & 337 & 29,88 & 5,98 & \multirow{2}{*}{$-1,875$} & \multirow{2}{*}{,061 } \\
\hline & No & 363 & 30,80 & 6,90 & & \\
\hline \multirow{2}{*}{ Hostility } & Yes & 337 & 27,20 & 5,41 & \multirow{2}{*}{$-1,165$} & \multirow{2}{*}{, 244} \\
\hline & No & 363 & 27,72 & 6,16 & & \\
\hline \multirow{2}{*}{ Anger } & Yes & 337 & 23,50 & 4,52 & \multirow{2}{*}{$-2,095$} & \multirow{2}{*}{,o37* } \\
\hline & No & 363 & 24,27 & 5,07 & & \\
\hline \multirow{2}{*}{ Verbal Aggression } & Yes & 337 & 16,83 & 3,47 & \multirow{2}{*}{$-1,813$} & \multirow{2}{*}{, 070} \\
\hline & No & 363 & 17,32 & 3,58 & & \\
\hline \multirow{2}{*}{ Total Aggression } & Yes & 337 & 93,71 & 16,60 & \multirow{2}{*}{$-2,195$} & \multirow{2}{*}{,o29* } \\
\hline & No & 363 & 96,71 & 18,59 & & \\
\hline
\end{tabular}

*p<0,05

As is seen in Table 12, since $p=0,037<0,049$, there is seen a significant difference $(p<0,05)$ in Anger sub-scale when looking at aggression levels sub-scales by the Smoking variable. A significant difference $(p<0,05)$ is found between aggression scores of aggression levels by the smoking variable. Since p>0,049 between Physical aggression, Hostility and Verbal aggression, there is no significant difference $(p>0,05)$.

Table-13. Significance Table of Aggression Levels Sub Scales by Alcohol Use Variable.

\begin{tabular}{|c|c|c|c|c|c|c|}
\hline Do you use alcohol? & & $\mathbf{n}$ & $\mathbf{x}$ & sd & $\mathbf{t}$ & $\mathbf{p}$ \\
\hline \multirow{2}{*}{ Physical Aggression } & Yes & 248 & 29,69 & 6,90 & \multirow{2}{*}{$-2,005$} & \multirow{2}{*}{, $045^{*}$} \\
\hline & No & 452 & 30,72 & 6,23 & & \\
\hline \multirow{2}{*}{ Hostility } & Yes & 248 & 26,84 & 6,16 & \multirow{2}{*}{$-2,109$} & \multirow{2}{*}{,035* } \\
\hline & No & 452 & 27,82 & 5,59 & & \\
\hline \multirow{2}{*}{ Anger } & Yes & 248 & 23,13 & 5,12 & \multirow{2}{*}{$-3,132$} & \multirow{2}{*}{, $002 *$} \\
\hline & No & 452 & 24,32 & 4,60 & & \\
\hline \multirow{2}{*}{ Verbal Aggression } & Yes & 248 & 16,83 & 3,76 & \multirow{2}{*}{$-1,420$} & \multirow{2}{*}{, 156} \\
\hline & No & 452 & 17,23 & 3,40 & & \\
\hline \multirow{2}{*}{ Total Aggression } & Yes & 248 & 92,52 & 18,99 & \multirow{2}{*}{$-2,957$} & \multirow{2}{*}{,003* } \\
\hline & No & 452 & 96,74 & 16,80 & & \\
\hline
\end{tabular}

Table-14. Significance Table of Aggression Levels Sub Scales by Income Level Variable

\begin{tabular}{|c|c|c|c|c|c|c|c|}
\hline Income Level & & n & $\mathbf{x}$ & sd & $f$ & p & Significance \\
\hline \multirow{4}{*}{ Physical Aggression } & Between 0-1000 & 174 & 27,98 & 7,81 & \multirow{4}{*}{20,827} & \multirow{4}{*}{,OOO* } & \multirow{4}{*}{$\begin{array}{l}\text { Between 0-1000- } 1000- \\
30000-1000-\text { more than } \\
30001000-3000-\text { over } \\
3000\end{array}$} \\
\hline & $1000-3000$ & 342 & 30,52 & 5,60 & & & \\
\hline & 3000 and over & 184 & 32,30 & 5,94 & & & \\
\hline & Total & 700 & 30,36 & 30,36 & & & \\
\hline \multirow{4}{*}{ Hostility } & Between 0-1000 & 174 & 25,57 & 6,64 & \multirow{4}{*}{20,585} & \multirow{4}{*}{,000* } & \multirow{4}{*}{$\begin{array}{l}\text { Between } \quad 0-1000 \\
1000-3000 \text { Between } \\
1000- \\
3000 \text {-over } 3000\end{array}$} \\
\hline & $1000-3000$ & 342 & 27,37 & 4,97 & & & \\
\hline & 3000 and over & 184 & 29,43 & 5,82 & & & \\
\hline & Total & 700 & 27,47 & 5,81 & & & \\
\hline \multirow{4}{*}{ Anger } & Between 0-1000 & 174 & 22,17 & 5,59 & \multirow{4}{*}{24,352} & \multirow{4}{*}{,OOO* } & \multirow{4}{*}{$\begin{array}{l}\text { O-1000-1000-3000 } \\
0-1000-\text { over } 30001000- \\
3000-\text { over } 3000\end{array}$} \\
\hline & $1000-3000$ & 342 & 23,84 & 4,15 & & & \\
\hline & 3000 and over & 184 & 25,63 & 4,64 & & & \\
\hline & Total & 700 & 23,90 & 4,82 & & & \\
\hline \multirow{4}{*}{ Verbal Aggression } & Between 0-1000 & 174 & 16,38 & 3,92 & \multirow{4}{*}{8,342} & \multirow{4}{*}{,000* } & \multirow{4}{*}{$\begin{array}{l}\text { Between 0-1000 - over } \\
3000-1000-3000 \text { - over } \\
3000\end{array}$} \\
\hline & $1000-3000$ & 342 & 17,01 & 3,16 & & & \\
\hline & 3000 and over & 184 & 17,89 & 3,66 & & & \\
\hline & Total & 700 & 17,09 & 3,53 & & & \\
\hline \multirow{4}{*}{ Total Aggression } & Between 0-1000 & 174 & 88,58 & 21,12 & \multirow{4}{*}{24,908} & \multirow{4}{*}{,OOO* } & \multirow{4}{*}{$\begin{array}{l}\text { Between 0-1000- } 1000- \\
3000 \\
\text { Between 0-1000- } 3000 \\
\text { and over } \\
1000-3000 \text {-over } 3000\end{array}$} \\
\hline & $1000-3000$ & 342 & 95,15 & 14,66 & & & \\
\hline & 3000 and over & 184 & 101,61 & 17,09 & & & \\
\hline & Total & 700 & 95,26 & 17,70 & & & \\
\hline
\end{tabular}


As is seen in Table 13, when looking at aggression levels sub-scales by 'Alcohol use' variable, since Physical aggression is $p=0,045<0,049$, Hostility is $p=0,035<0,049$ and Anger are $p=0,002<0,049$, there is found a significant difference $(\mathrm{p}<0,05)$ between these variables and alcohol use. Since $p>0,049$, there is no significant difference $(p>0,05)$ between verbal aggression and alcohol use.

As is seen in Table 14, there is found the difference between Physical Aggression, hostility, Verbal Aggression and Income levels as between 0-1000, between 1000-3000, 3000 and over.

\section{Discussion and Conclusion}

It is seen in our study that the aggression levels of male students are higher than the aggression levels of female students. Kırımoğlu et al. (2008) and Efilti (2008) found that the aggression levels of students in secondary education institutions are higher than those of female students. Scharf (2000) conducted a survey to determine the differences in aggression by gender. According to the findings, verbal aggression does not vary by gender; physical aggression is used more by males. Camadan and Yazıcı (2017) conducted a study called 'Analyzing the aggression tendencies of students regarding some of the variables.' At the end of the study, they found differences between aggression scores of female and male students. On the other hand, Yönet et al. (2016) conducted a research called 'Reviewing Aggression-Violence Tendencies of High School Students with Attendance to Recreative Activities.' In their findings, there is no significant difference between the aggression levels of students by the gender variable.

Giles and Heyman (2005) analyzed the relationship between gender and aggression in teenagers. They found that the aggression levels of males are higher than those of females. As is mentioned by Fromm (1993) testosterone increases aggression levels; while estrogen decreases aggression levels. Our study shows parallels with the literature.

Although there is no significant difference between the students studying in the faculty of sports sciences by gender, it is seen that the aggression levels of male students studying in the faculty of education and faculty of communication are higher in comparison with the aggression levels of female students. This result confirms the findings in Table 3 .

It is seen when the departments are compared that the aggression levels of the faculty of communication and faculty of education are higher than those of the students studying in the faculty of sport sciences. Sargin (2010) actualized a survey called 'Reviewing teacher candidates' awareness levels relating to conflict and violence by several variables'. Sargin analyzed the teacher candidates' awareness levels relating to conflict and violence by the department they study in. With reference to the findings, the conflict and violence awareness levels of teacher candidates in private areas are higher than the teacher candidates in numeric fields. Erşan et al. (2009) conducted a study called 'To evaluate the aggression levels of students in school of physical education and sports in terms of socio-demographic aspect'. According to the results of their study, there is no statistically significant difference in terms of department and branch. Erden (2007) examined the differences between the departments that the teacher candidates study in and the types of aggression. There is no significant difference between the departments. Yurttaş (2016) found that the point averages of students studying in the faculty of sport sciences are higher than the students studying in other faculties. As is understood above, there are different results in the literature. Moreover, it is thought within the scope of our research that the statuses, such as socio-demographic structure, personal differences, and region lived in, affected the aggression levels of the students studying in different departments.

We can say based upon our study that the children of authoritative parents have the highest aggression levels when looking at the physical aggression sub-scales by the family attitudes and total aggression scores. Under these circumstances, we are of the opinion that the children of domineering parents are more aggressive. Camadan and Yazıcı (2017) conducted a study named 'Reviewing the Aggression Tendencies Observed in University Students in terms of Several Variables'. According to the results of their study, the highest aggression levels belong to children who perceive the child-rearing method of their parents as authoritarian; the lowest aggression levels belong to children who perceive the child-rearing method of their parents as democratic. The aggression levels of university students statistically vary by the perception about the child rearing method of their parents. Yönet et al. (2016) conducted a survey named 'To examine the aggression-violence tendencies of high school students with their attendance to recreative activities'. With reference to their findings, there is a significant difference between the aggression levels of students by the family attitude variable.

It is seen that the aggression levels of students whose mothers are alive are higher than the aggression levels of students whose mothers are dead. However, there is no difference between the aggression scores of students whose fathers are alive and students whose fathers are dead. Moreover, we can point out that the students who lost their mothers are more aggressive than the students who lost their fathers. Erdogdu (2010) conducted a study called 'To analyze the aggression tendencies of students regarding different variables.' They found that the aggression tendencies significantly vary by the reaction status of their mother and father. The aggressive tendencies of students whose mother and father are dead are higher than those of students whose parents live together.

Per the results of our findings, the aggression levels of the students who do not play sports are higher in comparison with the students who play sports. Yıldız (2009) mentioned in his study that the people who play sports are more aggressive than the people who do not play sports on the disruptive aggression sub-scale; there is no statistically significant difference in the aggression sub-scale. Cobanoglu (1993) compared students who are athletes and students who are not athletes. He found that the aggressive tendencies of athletes are significantly higher than others. Kırımoğlu et al. (2008) and Dervent (2007) mentioned that males who play sports are more aggressive than males who do not play sports. However, there is no significant difference between them in other aggression features. Erdogdu (2010) and Sili (2012) conducted a survey 'to examine the aggression tendency of students regarding various variables.' They found that the aggression tendency of the students is associated with their regularly playing sports status. The aggressive tendencies of students who regularly play sport are significantly lower than those of students who do not play sports. Much as we monitor different results, the sporting activities increase the determination to win and aggression by force of the training and competitions. It 
can be thought that decreasing the aggression levels of people who play sports can be accepted as normal and foregone conclusions.

The aggression levels of students who do not smoke are higher than the students who smoke.

The aggression levels of students who do not use alcohol are higher than the students who use alcohol.

We can highlight in our study that people who have extended family are more aggressive, namely, as the number of people in the family increases, the aggression level increases at the same time. Factors such as being limited to meeting demands, discrepancies, dissidences and similar situations in extended families may increase the aggression.

According to the data of frustration-aggression hypothesis, when the relationship between socio-economic income level and aggression is analyzed, it is expected that the students who have a low-income status are more aggressive as a natural result of experiencing frustration because of economic insufficiencies (Kılınç, 2012). But, it is seen in our survey that as the income level increases, the level of aggression increases at the same time. Camadan and Yazıcı (2017); Sili (2012); Yilmaz (2008); Masalcı (2001) and Ağlamaz (2006) mention that as the income level decreases, the level of aggression increases. On the other hand, Kaynak (2013) expressed that increasing the income level affects the aggression level. According to the results of the study of Ece (2014) the general aggression scores of participants who have medium family income are lower than the attendees who have high family income. The conclusions of both the surveys confirm our study.

In conclusion, we can state that males are more aggressive than females; playing sport affects aggression levels; and people who do not play sports are more aggressive than people who play sports. It can be noted that this circumstance may stem from the characteristics of sports, such as renewing and discharging people. Much as there are different results in the literature, it is thought that aggression increases with the increase in income level. Moreover, the children of authoritative parents are more aggressive by the family attitude. It is also seen that students whose parents are dead are more aggressive in comparison with students whose parents are alive. Thus, lack of family love and an authoritarian attitude towards life may cause the aggression.

\section{References}

Acet, M., 2005. Aggression and violence in Sporda. Istanbul: Morpa Publications. pp: 55-56.

Ağlamaz, T., 2006. High school students' self - disclosure behavior of aggression score examination in terms of school type, gender, class level, parent education level and monthly income level. Master Thesis, 19th May University Social Sciences Institute, Samsun.

Asma, M., 2008. An examination of aggression perceptions of sportsmen participating in intercultural meetings in terms of social-cognitive learning theory (Case of Ankara Province). Gazi University Institute of Educational Sciences Department of Physical Education and Sports Teacher Education, (Unpublished Master Thesis).

Budak, S., 2000. Psychology dictionary. Ankara: Science and Art Publications.

Buss, A.H. and M. Perry, 1992. The aggression questionnaire. Journal of Personality and Social Psychology, 63(3): 452-459. View at Google Scholar

Camadan, F. and H. Yazıcı, 2017. Investigation of aggression trends observed in university students in terms of variable variables. Journal of Higher Education \& Science, 7(2): 225-234.

Cobanoglu, M.G., 1993. The effect of sporda aggression on sportive performance. İzmir, Dokuz Eylül University, Published Doctorate Thesis, 119.

Cox, R.H., 1985. Sport psychology. Dubuque, Iowa: Wm. C. Brown.

Creswell, J.W., 2012. Educational research: Planning, conducting, and evaluating quantitative and qualitative research. 4th Edn., Boston: Pearson.

Cüceloğlu, D., 2005. Human and behavior. Istanbul: Remzi Bookstore.

Dervent, F., 2007. Aggression levels of high school students and their relation to participation in sport activities. Gazi University Institute of Educational Sciences / Department of Physical Education and Sports Teacher Education, Master Thesis. Ankara.

Ece, C., 2014. Investigation of the aggressiveness levels of university students and secondary school students watching soccer competitions in the stadium (Sakarya Province). Graduate Thesis, Dumlupınar University Health Sciences Institute, Kütahya.

Efilti, E., 2008. A comparative study of aggression and control spaces of students reading in secondary education institutions. Doctoral Thesis, Institute of Social Sciences, Selcuk University, Konya.

Erden, N., 2007. Abant Izet Baysal University department of teaching first instruction types of aggression of last year students. Master Thesis, Abant Izzet Baysal University, Institute of Social Sciences, Bolu.

Erdogdu, M.Y., 2010. Students' aggression tendencies are examined in terms of some variables. International Conference on New Trends in Education and Their Implications. pp: 11-13.

Erşan, E., O. Doğan and S. Doğan, 2009. Socio-demographic evaluation of aggression levels of students of physical education and sport. Cumhuriyet University Cumhuriyet Journal of Medicine, 3 1(3): 231-238.

Fromm, E., 1993. Origins of destruction in the Inside. 2. Publication, Trans: Şükrü Alpagut, Istanbul: Payel Publishing House. pp: $38-68$.

Gergen, K.J. and M.N. Gergen, 1986. Social psychology. 2nd Edn., N.Y: Springer-Verlag.

Giles, J. and G.D. Heyman, 2005. Young children's beliefs about the relationship between genderand aggressive behavior. Child Development, 76(1): $107-121$. View at Google Scholar | View at Publisher

Güner, B., 2006. Examination of aggression levels of athletes engaged in team sports and individual sports. 19th May University of Health Sciences Institute Physical Education and Sports Department Master Degree Thesis.

Kaynak, A., 2013. The relationship between emotional intelligence levels and aggression levels of education faculty students is examined in terms of some variables. Graduate Thesis, Gaziantep University, Institute of Educational Sciences, Gaziantep.

Kılınç, E., 2012. Investigation of aggression levels of general high school 9th grade students according to some variables and trait anxiety levels. Graduate Thesis, Gaziantep University, Institute of Social Sciences, Gaziantep.

Kırımoğlu, H., N. Bright, C. Grade and A. Kepoğlu, 2008. Examination of high school students' aggression levels according to their level of participation in the spore. Niğde University Journal of Physical Education and Sport Sciences, 2(2): 147-154.

Kocacık, F., 2001. On the alleged violence, C.Ü. Journal of Economics and Administrative Sciences, 2(1): 1-7.

Köksal, F., 1991. Relations between control focus and aggressive behavior. Unpublished PhD Thesis, Ataturk University, Erzurum, Turkey.

Korkut, F., 2002. A preliminary study on the adaptation of the expressive aggression scale (ISÖ) to Turkish. Cukurova University Journal of Educational Sciences, 2(23): 48-53.

Kurtic, N., 2006. Psycho social causes attacking football surveillance. Master's Degree, Sakarya University Institute of Social Sciences Physical Education and Sports Teacher Education Department.

Leary, M.R., J.M. Twenge and E. Quinlivan, 2006. Interpersonal rejection as a determinant of anger and aggression. Personality and Social Psychology Review, 10(2): 111-132. View at Google Scholar $\mid$ View at Publisher

Madran, A.D., 2012. Validity and reliability study of Turkish form of Buss-Perry aggression scale. Journal of Turkish Psychiatry, 23 : 1-6.

Masalc1, D.A., 2001. Comparison of aggression level and appropriate behavior of the child by family interactions. Graduate Thesis, Dokuz Eylül University Educational Sciences Institute, Izmir.

Özdevecioğlu, M., 2003. A research on determining the impact of perceived organizational justice on individualistic aggressive behavior. Journal of Erciyes University Faculty of Economics and Administrative Sciences, 21: 77-96.

Özerkan, K.N., 2004. Introduction to sport psychology. Ankara: Nobel Publications. 
Russell, G.W., 2003. Sport riots: Social- psychological review, Canada foundations of sport. Champaign, IL: Human Kinetics Publishers

Sargin, N., 2010. Examination of teachers' awareness levels of conflict and severity according to various variables. Journal of Theory and Practice in Education Management, 16(4): 601-616.

Scharf, S.C., 2000. Gender differences in adolescent aggression: An analysis of instrumentality vs. Expressiveness. Unpublished Doctoral Thesis Michigan University Dep. of Clinical Psyc., Michigan.

Sili, A., 2012. A sociological assessment of high school students 'aggression tendencies: The sociological analysis of the secondary education students' aggressive behaviors. EKEV Akademi Magazine, 16: 261-271.

Star, S.A., 2004. Parental attitudes and aggression. Journal of Police Science, 6(3-4): 13 1-150.

Tiryaki, Ş., 2000. Sports psychology. Ankara: Publishing House. pp: 3-153.

Tok, Y., 2001. The level of aggression of university students with different pattern of judgments on gender roles. Unpublished Master's Thesis, Hacettepe University Social Sciences Institute.

Tutkun, E., B.Ç. Güner, S.A. Ağaoğlu and R. Soslu, 2010. Evaluation of aggressiveness levels of team sports and individual sports performers. Journal of Sport and Performance Studies, 1(1): 23-29.

Yetim, A., 2005. Sociology and sport. 3rd Edn., Istanbul: Morpa Culture Publications.

Yıldız, S., 2009. An investigation of aggressiveness levels of secondary school students who do and do not play. Graduate Thesis, Selcuk University Health Sciences Institute, Konya.

Yilmaz, İ., 2008. Multidimensional investigation of aggression levels of industrial vocational school students. Master Thesis, Yeditepe University Institute of Social Sciences, Istanbul.

Yönet, E., F. Çalik, F. Yaşartürk and K. Çimen, 2016. Investigation of aggression-violence trends by participation of high school students in recreational activities. International Journal of Science Culture and Sport, 4(3): 368-382. View at Google Scholar | View at Publisher

Yurttaş, H., 2016. An investigation of aggression levels of university students doing and doing sports. Graduate Thesis, Ataturk University Educational Sciences Institute, Erzurum.

\section{Bibliography}

Köknel, Ö., 1986. Personality to mutuality. Istanbul: Golden Books Publishing House.

Köknel Ö. 1986. Personality in mindful anxiety. Istanbul: Golden Book Publishing House. 